\title{
Characteristics of Cricopharyngeal Dysphagia After Ischemic Stroke
}

\author{
Hyuna Yang, MD, Youbin Yi, MD, Yong Han, MD, Hyun Jung Kim, MD, PhD
}

Department of Rehabilitation Medicine, Nowon Eulji Medical Center, Eulji University, Seoul, Korea

Objective To evaluate the characteristics of cricopharyngeal dysfunction (CPD), the frequency, and correlation with a brain lesion in patients with first-ever ischemic stroke, and to provide basic data for developing a therapeutic protocol for dysphagia management.

Methods We retrospectively reviewed the medical records of a series of subjects post-stroke who underwent a videofluoroscopic swallowing study (VFSS) from January 2009 to December 2015. VFSS images were recorded on videotape and analyzed. CPD was defined as the retention of more than $25 \%$ of residue in the pyriform sinus after swallowing. The location of the brain lesion was assessed using magnetic resonance imaging.

Results Among the 262 dysphagic patients with first-ever ischemic stroke, 15 (5.7\%) showed CPD on the VFSS. Patients with an infratentorial lesion had a significantly higher proportion of CPD than those with a supratentorial lesion ( $\mathrm{p}=0.003$ ), and lateral medullary infarction was identified as the single independent predictor of CPD (multivariable analysis: odds ratio=19.417; confidence interval, 5.560-67.804; $\mathrm{p}<0.0001$ ). Compared to patients without CPD, those with CPD had a significantly prolonged pharyngeal transit time, lower laryngeal elevation, and a higher pharyngeal constriction ratio and functional dysphagia scale score.

Conclusion Overall, the results support the notion that an impaired upper esopharyngeal opening is likely related to the specific locations of brain lesions. The association of CPD with lateral medullary infarction can be explained based on the regulation of the pharyngolaryngeal motor system by the motor neurons present in the dorsal nucleus ambiguus. Overall, the results reveal the relation between CPD and the problems in the pharyngeal phase as well as the severity of dysphagia.

Keywords Dysphagia, Cricopharyngeus, Upper esophageal sphincter, Stroke

\section{INTRODUCTION}

Swallowing dysfunction occurs as a result of neurologi- cal or mechanical disorders that impair the transport of food from the oral cavity to the stomach [1]. During the process of swallowing, the bolus passes through the

Received May 16, 2017; Accepted August 24, 2017

Corresponding author: Hyun Jung Kim

Department of Rehabilitation Medicine, Nowon Eulji Medical Center, Eulji University, 68 Hangeulbiseong-ro, Nowon-gu, Seoul 01830, Korea. Tel: +822-970-8315, Fax: +82-2-970-0028, E-mail: khj2603@eulji.ac.kr

ORCID: Hyuna Yang (http://orcid.org/0000-0002-2265-4166); Youbin Yi (http://orcid.org/0000-0001-5707-3815); Yong Han (http://orcid.org/00000001-5867-1227); Hyun Jung Kim (http://orcid.org/0000-0002-2198-5668).

(c) This is an open-access article distributed under the terms of the Creative Commons Attribution Non-Commercial License (http://creativecommons.org/ licenses/by-nc/4.0) which permits unrestricted noncommercial use, distribution, and reproduction in any medium, provided the original work is properly cited. Copyright $\odot 2018$ by Korean Academy of Rehabilitation Medicine 
upper esophageal sphincter (UES) and enters into the esophagus, which is located in the transition zone between the pharynx and the esophagus.

The UES is the term referring to the functional aspect of the pharyngoesophageal segment, which is comprised of the inferior pharyngeal constrictor, cricopharyngeus muscle, and proximal part of the cervical esophagus. Among these structures, the cricopharyngeus muscle is a main component of the UES, which acts as a muscular sling at the pharyngoesophageal junction and plays a vital role in the act of deglutition [2]. Normally, the cricopharyngeus muscle remains in a contracted position during respiration, preventing air from entering into the esophagus, and relaxes during swallowing when a bolus is pushed caudally by the tongue and the middle and inferior pharyngeal constrictor muscles [3]. Incoordination or hypertonicity of the cricopharyngeus muscle may lead to a range of symptoms, including dysphagia and aspiration $[2,4]$. Cricopharyngeal dysfunction (CPD) can be a consequence of various neurological and medical conditions such as cerebrovascular disease, Parkinson disease, amyotrophic lateral sclerosis, primary muscle disorders, esophageal diverticula, laryngectomy, skull base neoplasm, and muscular dysfunction [2]. However, the underlying mechanism of dysfunction has not been clearly determined yet [5].

CPD is considered to be caused by three main factors: (1) cricopharyngeus muscle spasm, (2) incomplete laryngeal movement, and (3) insufficient descending pressure of the bolus [6-8]. When CPD is caused solely by the cricopharyngeal muscle spasm, which inhibits the larynx from moving up and forward, then a botulinum toxin injection into the cricopharyngeus muscle or cricopharyngeal myotomy may be the treatment option after sufficient time is given for recovery $[5,9,10]$. The patients who exhibit reduced laryngeal movement will benefit from the Mendelsohn maneuver or Shaker exercise [11,12]. In case of weak pharyngeal contraction during swallowing, lingual exercises to improve tongue base action or effortful swallow may be helpful $[13,14]$.

The incidence of CPD is inconsistently reported in the literature. Most of the studies have been conducted in a heterogeneous patient population, and direct comparisons between the studies are difficult because of different study methods employed in the reported studies [2]. Among the stroke population, the incidence of CPD has been reported as high as $50 \%$ in patients with brainstem stroke, and the association between medullary infarction and CPD has been emphasized [15-17]. In addition, the development of a lesion after subcortical supratentorial strokes, such as basal ganglia or centrum semiovale infarction has been reported to cause CPD [6]. However, a large-scale study on CPD in patients with ischemic stroke has not been conducted yet. In addition, studies on lesion correlation and characteristics of CPD after stroke are sparse. Therefore, the objectives of this study were to investigate the incidence and characteristics of CPD according to the brain lesion location in patients with firstever ischemic stroke and to provide basic data for developing a therapeutic protocol for dysphagia management.

\section{MATERIALS AND METHODS}

\section{Subjects}

We retrospectively reviewed the medical records of a series of subjects post-stroke who underwent a videofluoroscopic swallowing study (VFSS) from January 2009 to December 2015. All the subjects who developed dysphagia after stroke were evaluated. Inclusion criteria were as follows: (1) patients older than 20 years; (2) those with first-ever ischemic stroke in which the brain lesion was verified by magnetic resonance imaging or computed tomography; and (3) those presenting with any symptoms or signs of dysphagia. Exclusion criteria were (1) patients with a previous history of any brain lesions such as stroke, traumatic brain injury, brain tumor, or Parkinson disease; (2) those with a previous history of dysphagia; and (3) those with any medical conditions or structural lesions that may affect swallowing function.

In total, 1,696 patients underwent VFSS during the study period. We excluded 1,429 patients according to the aforementioned criteria. Additionally, 5 patients were excluded because they were unable to initiate swallowing movement during the VFSS. Overall, 262 patients were included in the study (Fig. 1). This study was approved by the Institutional Review Board of Nowon Eulji Medical Center, Eulji University (No. EMCIRB 16-40).

\section{VFSS}

The VFSS was conducted by a rehabilitation physician in a videofluoroscopy room. Subjects were seated upright in a chair and swallowing images of the lateral projection 


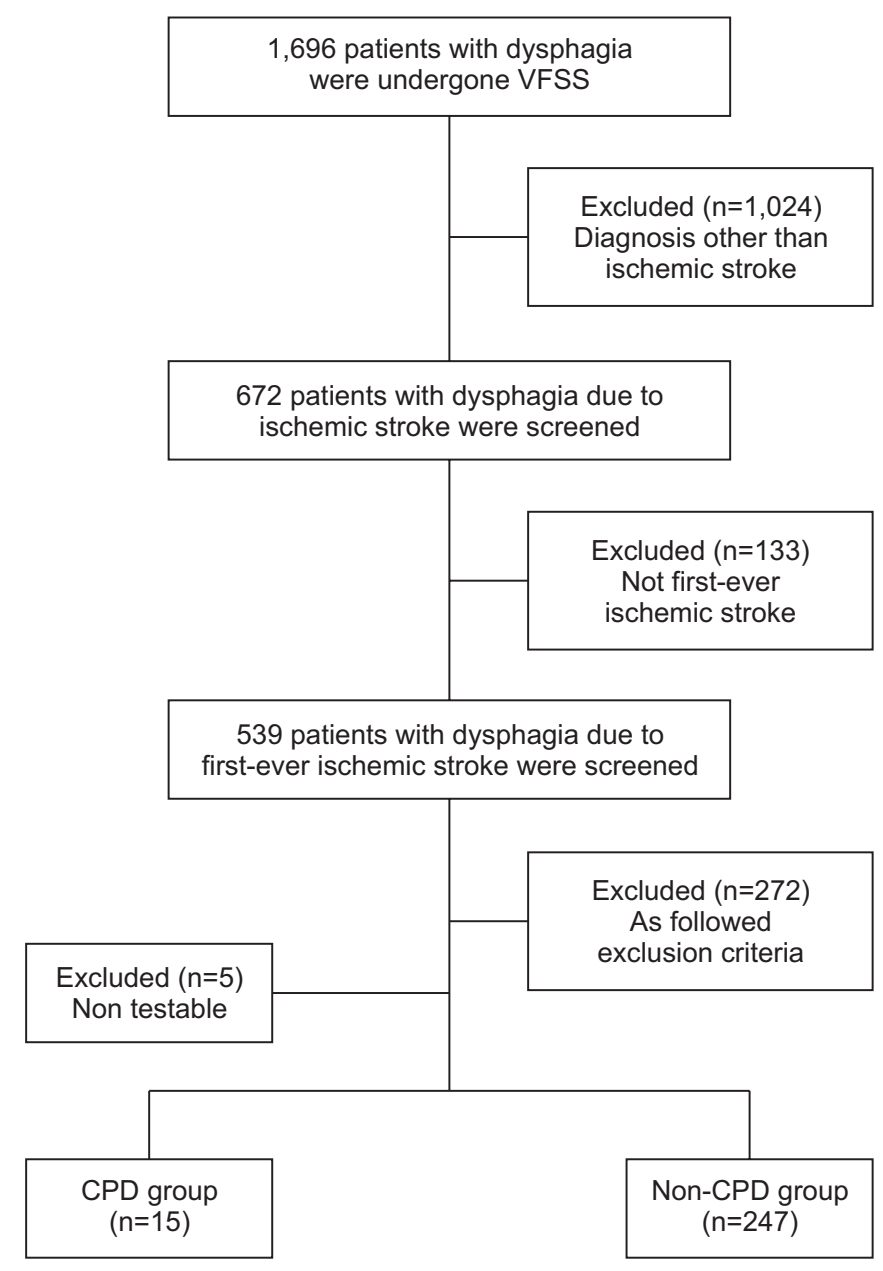

Fig. 1. Patient recruitment flow diagram detailing the number of evaluated and excluded subjects. VFSS, videofluoroscopic swallowing study; CPD, cricopharyngeal dysfunction.

were acquired (Fig. 2). Each subject was asked to swallow 2.5 and $5 \mathrm{~mL}$ each of thin rice gruel, rice porridge, rice, and liquid (as tolerated). The test was conducted based on the subject's general condition and status of dysphagia. Postural modification such as the chin tuck or head reclining was made when necessary. The test was terminated when massive evidence of airway compromise was observed. During the assessment, fluoroscopic images were simultaneously recorded using a digital camera (HMX-QF30; Samsung, Seoul, Korea) at 10 frames/s.

\section{Parameters}

The following parameters were assessed for each patient's swallowing process based on ingestion of liquid or puree. (1) Residue in the valleculae (VR) and pyriform

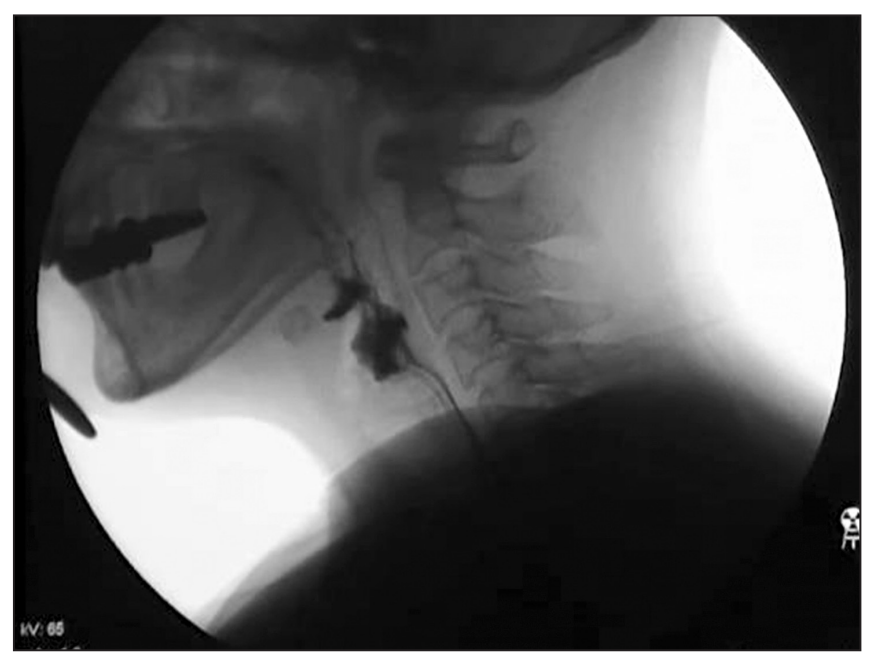

Fig. 2. Lateral still image taken during a videofluoroscopic swallowing study showing pyriform sinus and vallecular residue, with an impaired opening of the upper esophageal sphincter.

sinus (PSR), amounts were calculated as the percent of residue in the valleculae and pyriformis sinus and relative percentage of the pertinent area on a two-dimensional view $[6,18]$. (2) Oral transit time (OTT), defined as the duration between the first backward movement of the bolus to the time the head or tail of the bolus arrives at the point where the lower rim of the mandible crosses the tongue base (a normal value is considered 1.0-1.5 seconds or at most 1.75 seconds) [8,19]. (3) Pharyngeal delay time (PDT), defined as the time from when the bolus head arrives at the point where the lower edge of the mandible crosses the tongue base and ends when laryngeal elevation starts (in normal subjects, the PDT is minimal, and up to 0.5 seconds is acceptable for elderly patients older than 60 years) $[8,19]$. (4) Pharyngeal transit time (PTT) is the measurement of time from the initiation of PDT to the point where bolus tail passes through the cricopharyngeal region (values less than or equal to 1 second are indicative of an acceptable range) $[8,19]$. (5) The height of laryngeal elevation (LE) was measured from the first frame showing the upward movement of larynx to the last frame showing downward movement with the reference point at the posterior-superior corner of the subglottic air column [6]. The normal value is considered as $2 \mathrm{~cm}$ or more [6,8]. (6) Pharyngeal constriction ratio (PCR) is the ratio of pharyngeal area at maximal contraction to pharyngeal area at rest, which is considered as a surrogate measure for pharyngeal strength. The National 
Institutes of Health Image software (http://rsb.info.nih. gov/nih-image) was used for analysis. Spatial measurements were made after calibrating the digitized image to the size of a $2.2-\mathrm{cm}$-diameter radiopaque disc taped to the chin of the study subject [20]. Leonard et al. [20] reported $0.03 \pm 0.02$ as the mean value of PCR for a $3-\mathrm{mL}$ liquid bolus. (7) Functional dysphagia scale (FDS), which is designed to assess dysphagia severity. This scale was developed to evaluate dysphagia in stroke patients, and is comprised of 11 factors, including the overall swallowing process from the mouth to the pharynx. The summated FDS score ranges from 0 to 100 [18]. The presence of CPD was defined when the PSR was more than $25 \%$ after swallowing $[6,18]$. None of the subjects had anatomical changes, such as a diverticulum, and there was no evidence of relaxation disorders of the UES $[6,8]$.

\section{Brain lesion classification}

We classified the location of brain lesion in patients based on the method of Steinhagen et al. [17] as follows: frontal infarction, parietotemporal infarction, infarction of the basal ganglia or capsula interna, pontine infarction, lateral medullary infarction, and cerebellar infarction. We excluded the category involving the middle cerebral artery or pontine infarction affecting the upper motor neurons of the cranial nerves because the lesions of this category overlap with the lesions of other categories.

\section{Statistical analysis}

Patients were classified into the CPD group or nonCPD group according to the findings of the video analysis. Comparisons of demographic characteristics and clinical data were made between the CPD and non-CPD groups. For these comparisons, the independent-sample t-test, and Mann-Whitney U-test were used according to the types of variables, as appropriate. All the relevant covariates of the lesion location with a p-value $<0.1$ were

Table 1. Characteristics and locations of lesions in the CPD group versus non-CPD group

\begin{tabular}{|c|c|c|c|}
\hline Variable & CPD group $(n=15)$ & Non-CPD group $(n=247)$ & p-value \\
\hline Age (yr) & $71.07 \pm 10.65$ & $71.85 \pm 12.55$ & 0.789 \\
\hline Sex & & & 0.193 \\
\hline Male & 10 & 121 & \\
\hline Female & 5 & 126 & \\
\hline Onset to study (day) & $21.60 \pm 18.45$ & $25.71 \pm 76.32$ & 0.835 \\
\hline \multicolumn{4}{|l|}{ Lesion location_1 } \\
\hline FRI & $4(1)$ & $104(11)$ & 0.280 \\
\hline PTI & 4 & $113(6)$ & $0.088^{*}$ \\
\hline BCI & 3 & $60(44)$ & $0.078^{*}$ \\
\hline POI & $1(2)$ & $9(33)$ & 0.800 \\
\hline LMI & $0(6)$ & $2(6)$ & $<0.001^{*}$ \\
\hline Cbll & 2 & $16(8)$ & 0.675 \\
\hline Other ${ }^{\text {a) }}$ & 0 & 6 & \\
\hline \multicolumn{4}{|l|}{ Lesion location_2 } \\
\hline Supratentorial lesion & 5 & 174 & $0.003^{* *}$ \\
\hline Infratentorial lesion & 9 & 54 & \\
\hline Both lesion & 1 & 19 & \\
\hline
\end{tabular}

Values are presented as mean \pm standard deviation, and numbers in parentheses indicate number of patients with combined (isolated) infarction in the referring brain region.

CPD, cricopharyngeal dysfunction; FRI, frontal infarction; PTI, parietotemporal infarction; BCI, infarction of basal ganglia or capsula interna; POI, pontine infarction; LMI, lateral medullary infarction; Cbll, cerebellar infarction.

${ }^{a)}$ Lesion location, which was not classified by Steinhagen's method, was counted. Three patients with thalamic infarction and three patients with medial medullary lesion were included.

*p $<0.1$ Mann-Whitney test.

${ }^{* *} \mathrm{p}<0.01$ based on chi-square test. 
entered into the multivariate model following univariate analysis. Results are presented as odds ratios with $95 \%$ confidence intervals. In addition, patients were divided into three groups based on the location of lesions as supratentorial lesion, infratentorial lesion, and both the lesions for further analysis. The chi-square test was used to test the presence of significant differences among the three groups. Analyses were performed using IBM SPSS Statistics version 22.0 for Windows (IBM, Armonk, NY, USA).

\section{RESULTS}

Demographic characteristics and distribution of the stroke lesion location are demonstrated in Table 1. The mean age of the patients in the CPD group and the nonCPD group was $71.07 \pm 10.65$ years and $71.85 \pm 12.55$ years, respectively. Among the 262 dysphagic patients with firstever ischemic stroke, 15 (5.7\%) showed CPD on the VFSS. When all the subjects were further classified based on the location of the lesions, 5 patients $(2.8 \%)$ had supratentorial lesions, 9 patients (14.3\%) had infratentorial lesions, and 1 patient (5.0\%) had both the lesions with CPD. A significant difference was found in the frequency of CPD according to the location of the lesions. Patients with infratentorial lesions had a significantly higher proportion of CPD than those with supratentorial lesions ( $\mathrm{p}=0.003)$.

In univariate analysis, lateral medullary infarction, infarction of the basal ganglia or internal capsule, and parietotemporal infarction were selected as relevant parameters $(\mathrm{p}<0.1)$. All the relevant parameters from univariate analysis were adjusted in multivariate analysis (Table 2). Lateral medullary infarction was identified as the single independent predictor of CPD (odds ratio $=19.417$; 95\% confidence interval, 5.560-67.804; $\mathrm{p}<0.0001)$.

Table 2. Summary of results of the multivariate logistic regression model for predicting high-risk CPD

\begin{tabular}{ccc}
\hline Lesion location & OR (95\% CI) & p-value \\
\hline PTI & $0.373(0.115-1.203)$ & 0.099 \\
BCI & $0.335(0.092-1.218)$ & 0.097 \\
LMI & $19.417(5.560-67.804)$ & $<0.0001^{*}$ \\
\hline
\end{tabular}

CPD, cricopharyngeal dysfunction; OR, odds ratio; CI, confidence interval; PTI, parietotemporal infarction; BCI, infarction of basal ganglia or capsula interna; LMI, lateral medullary infarction.

${ }^{*} \mathrm{p}<0.0001$.
Results of the VFSS, including the OTT, PDT, PTT, LE, PCR, and FDS, were compared between the two patient groups (Table 3). Patients in the CPD group had a significantly prolonged PTT, lower LE, and higher PCR and FDS than those in the non-CPD group $(\mathrm{p}<0.001, \mathrm{p}=0.036$, $\mathrm{p}<0.001$, and $\mathrm{p}<0.001$, respectively). The PDT was marginally prolonged in the CPD group compared to the nonCPD group $(\mathrm{p}=0.059)$.

Pearson correlation analysis was used to identify the factor exhibiting a significant relationship with PSR, which is a diagnostic factor of CPD. A high correlation was observed between the PCR and PSR (correlation coefficient $=0.894$, $\mathrm{p}<0.001$ ); all the values, except OTT, were significant, and the Pearson coefficients for the PTT, PDT, LE, and FDS were $<0.5$, indicating a low correlation (correlation coeffi-

Table 3. Comparison of swallowing functions between the two groups with and without CPD

\begin{tabular}{lccc}
\hline Variable & $\begin{array}{c}\text { CPD group } \\
(\mathbf{n}=15)\end{array}$ & $\begin{array}{c}\text { Non-CPD group } \\
(\mathbf{n}=\mathbf{2 4 7})\end{array}$ & p-value \\
\hline OTT (s) & $0.94 \pm 0.41$ & $1.12 \pm 0.83$ & 0.416 \\
PDT (s) & $1.73 \pm 1.46$ & $0.96 \pm 0.65$ & 0.059 \\
PTT (s) & $2.78 \pm 2.01$ & $1.24 \pm 0.70$ & $<0.001^{* *}$ \\
LE (cm) & $1.63 \pm 0.47$ & $1.95 \pm 0.46$ & $0.036^{*}$ \\
PCR & $0.15 \pm 0.03$ & $0.04 \pm 0.01$ & $<0.001^{* *}$ \\
FDS (score) & $45.07 \pm 13.83$ & $20.51 \pm 16.42$ & $<0.001^{* *}$ \\
\hline
\end{tabular}

Values are presented as mean \pm standard deviation.

CPD, cricopharyngeal dysfunction; OTT, oral transit time; PDT, pharyngeal delay time; PTT, pharyngeal transit time; LE, laryngeal elevation; PCR, pharyngeal constriction ratio; FDS, functional dysphagia scale.

${ }^{*} \mathrm{p}<0.05,{ }^{* *} \mathrm{p}<0.001$.

Table 4. Results of correlation analysis of parameters associated with PSRs

\begin{tabular}{lcr}
\hline & Pearson coefficient (rho) & p-value \\
\hline PSR & 1.000 & \\
OTT & -0.035 & 0.568 \\
PDT & 0.396 & $<0.001$ \\
PTT & 0.273 & $<0.001$ \\
LE & -0.207 & 0.001 \\
PCR & 0.894 & $<0.001$ \\
\hline FDS & 0.343 & $<0.001$ \\
\hline
\end{tabular}

PSR, pyriform sinus residue; OTT, oral transit time; PDT, pharyngeal delay time; PTT, pharyngeal transit time; LE, laryngeal elevation; PCR, pharyngeal constriction ratio; FDS, functional dysphagia scale. 
cient $=0.273,0.396,-0.207$, and 0.343 , respectively) (Table 4).

Table 5 shows a summary of the VFSS findings of patients in the CPD group. Compared to normal values, patients with CPD had an acceptable OTT. Thirteen out of 15 patients had a prolonged PTT. Except for 2 patients with a mild delay of the PDT (i.e., 0.6 seconds), 13 had a considerable delay of the PDT. Laryngeal elevation was impaired in 10 patients. The PCR was considerably higher in all the patients with CPD. Among the CPD patients, PCR in lateral medullary groups was significantly higher compared to the patients with stroke lesion other than lateral medulla $(\mathrm{p}=0.041)$.

\section{DISCUSSION}

The overall incidence of CPD in the present study population with first-ever ischemic stroke with dysphagia was $5.7 \%$. Among the patients with the infratentorial le- sion, the incidence of CPD was $14.3 \%$ and was found in $2.8 \%$ of patients with supratentorial lesions. One of the remarkable findings of this study is that supratentorial lesions after ischemic stroke can also cause CPD. Until recently, findings of an impaired UES opening by lateral medullary infarction have received attention in brainstem infarction. However, a study reported that stroke lesions of supratentorial subcortical structures lead to significant pharyngeal symptoms such as pharyngeal delay and a reduced cricopharyngeal opening [6]. In addition, alterations in cricopharyngeus function have been reported in certain neurological disorders such as Parkinson disease and amyotrophic lateral sclerosis [21-24]. Multifactorial mechanisms involving supramedullary as well as medullary function have been accounted as underlying mechanisms in CPD, as the former is associated with the disease, and the role of the latter is explained as an association between progressive degeneration of the

Table 5. Findings of the videofluoroscopic swallowing study in patients with cricopharyngeal dysfunction

\begin{tabular}{|c|c|c|c|c|c|c|c|c|c|c|}
\hline No. & Lesion location & $\begin{array}{l}\text { OTT } \\
(\mathbf{s})\end{array}$ & $\begin{array}{l}\text { PDT } \\
(\mathrm{s})\end{array}$ & $\begin{array}{c}\text { PTT } \\
(\mathbf{s})\end{array}$ & $\begin{array}{l}\text { Laryngeal } \\
\text { elevation } \\
\text { (cm) }\end{array}$ & PCR & $\begin{array}{l}\text { Vallecular } \\
\text { residue } \\
\text { (grade) }\end{array}$ & $\begin{array}{l}\text { Pyriform } \\
\text { residue }^{\text {a) }} \\
\text { (grade) }\end{array}$ & FDS & $\begin{array}{l}\text { Airway } \\
\text { compro- } \\
\text { mise }\end{array}$ \\
\hline 1 & $\begin{array}{l}\text { Lt. pons, bilateral cerebel- } \\
\text { lum }\end{array}$ & 1.3 & 0.6 & 1.5 & 2.1 & 0.107 & 2 & 2 & 28 & Yes \\
\hline 2 & $\begin{array}{l}\text { Lt. frontal, parietal lobe, } \\
\text { bilateral cerebellum }\end{array}$ & 1.1 & 1.2 & 1.5 & 2.0 & 0.113 & 1 & 2 & 60 & Yes \\
\hline 3 & Lt. pons & 1.4 & 1.0 & 1.1 & 0.9 & 0.130 & 1 & 2 & 24 & No \\
\hline 4 & $\begin{array}{l}\text { Rt. frontal, parietal lobe, } \\
\text { basal ganglia, corona } \\
\text { radiata }\end{array}$ & 1.6 & 1.1 & 5.8 & 0.9 & 0.138 & 1 & 2 & 58 & Yes \\
\hline 5 & Lt. frontal, parietal lobe & 0.7 & 2.0 & 2.3 & 1.1 & 0.143 & 1 & 2 & 54 & No \\
\hline 6 & Lt. lateral medulla & 0.7 & 0.9 & 1.0 & 2.0 & 0.147 & 1 & 2 & 48 & Yes \\
\hline 7 & Rt. lateral medulla & 1.0 & 0.6 & 0.8 & 2.0 & 0.149 & 1 & 2 & 26 & No \\
\hline 8 & $\begin{array}{l}\text { Lt. basal ganglia, corona } \\
\text { radiata }\end{array}$ & 1.1 & 1.5 & 1.6 & 2.0 & 0.149 & 1 & 2 & 58 & No \\
\hline 9 & Lt. frontal lobe & 0.4 & 1.4 & 5.8 & 1.9 & 0.151 & 2 & 2 & 62 & Yes \\
\hline 10 & Lt. pons & 0.1 & 1.3 & 2.0 & 1.0 & 0.159 & 2 & 2 & 24 & Yes \\
\hline 11 & Lt. lateral medulla & 0.6 & 1.0 & 1.8 & 1.8 & 0.166 & 1 & 2 & 36 & No \\
\hline 12 & Rt. lateral medulla & 1.1 & 2.7 & 3.1 & 1.8 & 0.181 & 1 & 3 & 47 & No \\
\hline 13 & Rt. lateral medulla & 0.6 & 6.5 & 7.3 & 1.9 & 0.183 & 1 & 3 & 51 & Yes \\
\hline 14 & Rt. lateral medulla & 1.2 & 1.8 & 2.3 & 1.1 & 0.186 & 2 & 3 & 46 & Yes \\
\hline 15 & Lt. parietal lobe & 1.2 & 2.5 & 3.0 & 1.9 & 0.201 & 2 & 2 & 54 & Yes \\
\hline
\end{tabular}

OTT, oral transit time; PDT, pharyngeal delay time; PTT, pharyngeal transit time; PCR, pharyngeal constriction ratio; FDS, functional dysphagia scale.

${ }^{\text {a) }}$ Residue Grade 1=mild 25\%-50\% remained relative to the total bolus; Grade $2=$ moderate $50 \%-75 \%$; Grade $3=$ severe $\geq 75 \%$ accordingly. 
excitatory and inhibitory corticobulbar pyramidal fibers [21-24].

The central pattern generator (CPG), which is one of the main centers of swallowing control, is located in the medulla within the nucleus tractus solitarius and in the adjacent reticular formation [1]. The triggering of swallowing and control of motor neurons is regulated by the CPG. Therefore, if infarction occurs in the medulla, the swallowing function may be widely affected because of a disconnection syndrome with the contralateral CPG.

At the cortical level, primary motor, supplementary motor, and primary somatosensory area have been implicated in swallowing motor control. It is presumed that these areas play an important role in the beginning of the voluntary oral phase and pharyngeal phase [25]. Actually, transcranial magnetic stimulation applied to the motor cortex can produce motor evoked potential on the cricopharyngeal muscle and elicit contraction of the UES $[26,27]$. Kahrilas et al. [28] demonstrated that the width of the UES opening depends on the volume of the passing bolus material. This finding supports the suggestion that afferent information modulates swallowing movement and that modulatory effects have cortical mechanisms, signifying that the precentral cortex receives somatosensory inputs through the somatosensory cortex and thalamocortical fiber [25]. Subcortical and supratentorial lesions could damage the corticobulbar tract or extrapyramidal system (the circuit of the cortex-neostriatumdorsal thalamus-cortex, and loop of neostriatum-nigra), thereby causing impairment of the suprasegmental inhibitory effects on the bulbar center [6]. Consequently, the cricopharyngeus sphincter reflex pattern gets disinhibited and induce the hyperreflexic nature of cricopharyngeus sphincter [29]. However, a comprehensive model of the complex connection between supratentorial and bulbar structures is still lacking. In this study, classification of the cortex was based on the location of traditional anatomical lesions. The size and location of each tract that may affect swallowing function were not considered in the present study, therefore limiting the discovery of any association between specific brain lesion and CPD.

In this study, the CPD group had a significantly prolonged PTT, lower LE, and higher PCR and FDS than the non-CPD group. These findings indicate that the CPD group had a further slowing down of the pharyngeal phase, weakened pharyngeal construction, reduction in the laryngeal elevation, and overall lower function compared to the non-CPD group. The significant relationship of CPD with the FDS score signifies that CPD is associated with the overall parameters of dysphagia. A high FDS score suggests that the patient has severe dysphagia and requires rapid intervention.

Our findings of a lower LE in the CPD group are explained based on the results from previous studies conducted using electromyography in which the UES opening was elicited by coordination between laryngeal elevation and cricopharyngeus relaxation [30,31]. Additionally, our results showed that the PDT was marginally significant in CPD group compared to the non-CPD group; thus, it can be considered that a voluntary trigger using a purely reflex mechanism of the bulbar swallowing center may work before the initiation of severe degeneration of the corticobulbar-pyramidal motor tract [24].

Overall, patients with CPD had significantly higher PCR values. Since the PCR is considered a surrogate measure for pharyngeal strength, a higher PCR value reveals ineffectiveness of the pharyngeal function during pharyngeal clearing or bolus propulsion [20]. Considering that CPD occurs in relation with the cricopharyngeus opening, laryngohyoid elevation, and pharyngeal contraction, we expect the occurrence of residue because of dysfunction of the pharyngeal segment rather than a single problem associated with the cricopharyngeus proper [9]. In addition, patients with CPD with lateral medullary lesions exhibited a higher constriction ratio, which indicates that lateral medullary lesions have a significant effect on the pharyngeal segment strength in those with CPD. Moreover, this finding was statistically significant when stroke lesions in lateral medullary groups were compared to the patients with stroke lesions in areas other than the lateral medulla ( $\mathrm{p}=0.041$ ).

Furthermore, the high correlation between the PSR and PCR demonstrated that pharyngeal strength had the greatest effect on residue, which is supported by the results from previous studies in patients with Parkinson. A study conducted by Ali et al. [22] showed that incomplete UES relaxation measured by manometry could be sufficiently compensated by the pharyngeal propulsive and clearance mechanism, thus overcoming the effect of the non-relaxing UES. Effortful swallow for pharyngeal strengthening, isometric lingual exercise for increasing intrabolus pressure, and the Shaker exercise for increas- 
ing UES opening by increasing hyolaryngeal excursion have been proven to be helpful for pharyngeal dysphagia $[12,13]$.

Studies on the long-term prognosis of CPD as well as the systematic protocol for the management of CPD are inadequate. If CPD is present, early intervention to prevent changes in the pharyngeal dimension caused by weakness is required to avoid irreversible pharyngeal dilatation and weakness [32]. Therefore, it is hypothesized that the early diagnosis of CPD, according to the lesion location of the stroke, and appropriate management strategy will contribute to improving the patients' quality of life and survival of dysphagic patients with CPD.

There are several limitations in this study. First, we primarily assessed swallowing function with the VFSS and measured pharyngeal strength by calculating the PCR. Although the VFSS is the gold standard method for evaluating swallowing function, manometry can provide more accurate information regarding pharyngeal and esophageal pressure events during the swallowing process. Therefore, further evaluation of swallowing function by pairing fluoroscopy with manometry is warranted. Second, we did not consider the infarct size. The threedimensional extent of the lesion needs to be considered in the future. Third, our findings are derived from a retrospective study design; thereby, a risk of bias from any confounders exists despite our effort. Fourth, there was a relatively small sample size in the CPD group, so it was difficult to delineate the characteristics of the CPD group. Lastly, in our study, none of the patients had true cricopharyngeus muscle dysfunction such as cricopharyngeal muscle achalasia. This may be the specific characteristic of stroke patients, but it was not observed in our study because of the small sample size. In the future, further large-scale studies are required to address this issue.

In conclusion, the results support the notion that an impaired UES opening is likely related to the specific brain lesion locations. Additionally, CPD is related to problems in the pharyngeal phase and severity of dysphagia. Findings of an association between lateral medullary infarction and CPD are in agreement with the results from previous reports. CPD associated with lateral medullary infarction can be explained by the effect on the dorsal nucleus ambiguus that contains premotor neurons, which regulate the pharyngolaryngeal motor system. In addition, damage to the corticobulbar tract or extrapyramidal system can cause CPD. Further studies are needed to elucidate the pathophysiological mechanism of CPD.

\section{CONFLICT OF INTEREST}

No potential conflict of interest relevant to this article was reported.

\section{REFERENCES}

1. Gonzalez-Fernandez M, Daniels SK. Dysphagia in stroke and neurologic disease. Phys Med Rehabil Clin N Am 2008;19:867-88.

2. Baredes S, Shah CS, Kaufman R. The frequency of cricopharyngeal dysfunction on videofluoroscopic swallowing studies in patients with dysphagia. Am J Otolaryngol 1997;18:185-9.

3. Lang IM. Brain stem control of the phases of swallowing. Dysphagia 2009;24:333-48.

4. Dou Z, Zu Y, Wen H, Wan G, Jiang L, Hu Y. The effect of different catheter balloon dilatation modes on cricopharyngeal dysfunction in patients with dysphagia. Dysphagia 2012;27:514-20.

5. Kocdor P, Siegel ER, Tulunay-Ugur OE. Cricopharyngeal dysfunction: a systematic review comparing outcomes of dilatation, botulinum toxin injection, and myotomy. Laryngoscope 2016;126:135-41.

6. Wan P, Chen X, Zhu L, Xu S, Huang L, Li X, et al. Dysphagia post subcortical and supratentorial stroke. J Stroke Cerebrovasc Dis 2016;25:74-82.

7. Fager SK, Hakel M, Brady S, Barlow SM, Nordness A, Delgado A, et al. Adult neurogenic communication and swallowing disorders. In: Cifu DX, editor. Braddom's physical medicine and rehabilitation. 5th ed. Philadelphia: Elsevier; 2016. p. 53-69.

8. Logemann JA. Evaluation and treatment of swallowing disorders. Austin: Pro-Ed; 1995.

9. Kuhn MA, Belafsky PC. Management of cricopharyngeus muscle dysfunction. Otolaryngol Clin North Am 2013;46:1087-99.

10. Lee SY, Seo HG, Paik NJ. Botulinum toxin injection for dysphagia: a blinded retrospective videofluoroscopic swallowing study analysis. Am J Phys Med Rehabil 2009;88:491-4.

11. Kahrilas PJ, Logemann JA, Lin S, Ergun GA. Pharyn- 
geal clearance during swallowing: a combined manometric and videofluoroscopic study. Gastroenterology 1992;103:128-36.

12. Shaker R, Easterling C, Kern M, Nitschke T, Massey B, Daniels $\mathrm{S}$, et al. Rehabilitation of swallowing by exercise in tube-fed patients with pharyngeal dysphagia secondary to abnormal UES opening. Gastroenterology 2002;122:1314-21.

13. Robbins J, Kays SA, Gangnon RE, Hind JA, Hewitt $A L$, Gentry LR, et al. The effects of lingual exercise in stroke patients with dysphagia. Arch Phys Med Rehabil 2007;88:150-8.

14. Steele CM, Huckabee ML. The influence of orolingual pressure on the timing of pharyngeal pressure events. Dysphagia 2007;22:30-6.

15. Lee CK, Kim JA. Pattern of post-stroke swallowing disorder according to the brain lesion. J Korean Acad Rehabil Med 2001;25:193-201.

16. Bian RX, Choi IS, Kim JH, Han JY, Lee SG. Impaired opening of the upper esophageal sphincter in patients with medullary infarctions. Dysphagia 2009;24:23845.

17. Steinhagen V, Grossmann A, Benecke R, Walter U. Swallowing disturbance pattern relates to brain lesion location in acute stroke patients. Stroke 2009;40:19036.

18. Han TR, Paik NJ, Park JW. Quantifying swallowing function after stroke: a functional dysphagia scale based on videofluoroscopic studies. Arch Phys Med Rehabil 2001;82:677-82.

19. Rademaker AW, Pauloski BR, Logemann JA, Shanahan TK. Oropharyngeal swallow efficiency as a representative measure of swallowing function. J Speech Hear Res 1994;37:314-25.

20. Leonard RJ, Kendall KA, McKenzie S, Goncalves MI, Walker A. Structural displacements in normal swallowing: a videofluoroscopic study. Dysphagia 2000;15: 146-52.

21. Alfonsi E, Versino M, Merlo IM, Pacchetti C, Martignoni $\mathrm{E}$, Bertino $\mathrm{G}$, et al. Electrophysiologic patterns of oral-pharyngeal swallowing in parkinsonian syndromes. Neurology 2007;68:583-9.
22. Ali GN, Wallace KL, Schwartz R, DeCarle DJ, Zagami AS, Cook IJ. Mechanisms of oral-pharyngeal dysphagia in patients with Parkinson's disease. Gastroenterology 1996;110:383-92.

23. Mu L, Sobotka S, Chen J, Su H, Sanders I, Adler CH, et al. Altered pharyngeal muscles in Parkinson disease. J Neuropathol Exp Neurol 2012;71:520-30.

24. Ertekin C, Aydogdu I, Yuceyar N, Kiylioglu N, Tarlaci S, Uludag B. Pathophysiological mechanisms of oropharyngeal dysphagia in amyotrophic lateral sclerosis. Brain 2000;123(Pt 1):125-40.

25. Martin RE, Sessle BJ. The role of the cerebral cortex in swallowing. Dysphagia 1993;8:195-202.

26. Ertekin C, Turman B, Tarlaci S, Celik M, Aydogdu I, Secil Y, et al. Cricopharyngeal sphincter muscle responses to transcranial magnetic stimulation in normal subjects and in patients with dysphagia. Clin Neurophysiol 2001;112:86-94.

27. Valdez DT, Salapatek A, Niznik G, Linden RD, Diamant NE. Swallowing and upper esophageal sphincter contraction with transcranial magnetic-induced electrical stimulation. Am J Physiol 1993;264(2 Pt 1):G2139.

28. Kahrilas PJ, Dodds WJ, Dent J, Logemann JA, Shaker R. Upper esophageal sphincter function during deglutition. Gastroenterology 1988;95:52-62.

29. Ertekin C, Aydogdu I, Tarlaci S, Turman AB, Kiylioglu N. Mechanisms of dysphagia in suprabulbar palsy with lacunar infarct. Stroke 2000;31:1370-6.

30. Perlman AL, Palmer PM, McCulloch TM, Vandaele DJ. Electromyographic activity from human laryngeal, pharyngeal, and submental muscles during swallowing. J Appl Physiol (1985) 1999;86:1663-9.

31. Venker-van Haagen AJ, Hartman W, van den Brom WE, Wolvekamp WT. Continuous electromyographic recordings of pharyngeal muscle activity in normal and previously denervated muscles in dogs. Am J Vet Res 1989;50:1725-8.

32. Arenaz Bua B, Olsson R, Westin U, Rydell R, Ekberg O. Treatment of cricopharyngeal dysfunction: a comparative pilot study. BMC Res Notes 2015;8:301. 